Molecules 2004, 9, 1222-1235

molecules

ISSN 1420-3049

http://www.mdpi.org

\title{
Valence Topological Charge-Transfer Indices for Dipole Moments: Percutaneous Enhancers
}

\section{Francisco Torrens *}

Institut Universitari de Ciència Molecular, Universitat de València, Dr. Moliner 50, E-46100 Burjassot (València), Spain. http://www.uv.es/ icmol. Tel. (+34) 963543 182; Fax (+34) 963543156.

* Tho whom correspondence should be addressed: E-mail: Francisco.Torrens@uv.es

Received: 2 June 2004 / Accepted: 18 June 2004 / Published: 31 December 2004

\begin{abstract}
Valence topological charge-transfer (CT) indices are applied to the calculation of dipole moments. The algebraic and vector semisum CT indices are defined. The combination of CT indices allows the estimation of the dipole moments. The model is generalized for molecules with heteroatoms. The ability of the indices for the description of the molecular charge distribution is established by comparing them with the dipole moments of homologous series of percutaneous enhancers (phenyl alcohols and 4-alkylanilines). Linear and quadratic correlation models are obtained. CT indices improve the multivariable quadratic regression equations for the dipole moment. The variance decreases $97 \%$ (4-alkylanilines). No superposition of the corresponding $G_{k}-J_{k}$ and $G_{k}{ }^{V}-J_{k}{ }^{V}$ pairs is observed in the fits, which diminishes the risk of co-linearity. The inclusion of the heteroatom in the $\pi$-electron system is beneficial for the description of the dipole moment, owing to either the role of the additional $p$ orbitals provided by the heteroatom or the role of steric factors in the $\pi$-electron conjugation. Inclusion of a conjugated double bond in the alkyl chain lends to more rigid structures with dipole moment variations $<1 \%$.
\end{abstract}

Keywords: Transdermal drug delivery, percutaneous enhancer, charge distribution, dipole moment, valence topological charge-transfer index.

\section{Introduction}

Passive drug penetration across absorbent membranes involves both the intrinsic resistance of the lipidic barriers themselves and that of the adjacent aqueous boundary layers, which can provide 
substantial additional strength against the passage of penetrants [1,2]. When adjacent to the lipoidal membrane and its luminal side, aqueous boundary layer thickness can be modified by agitation and, for in vitro experiments, the importance of suitable stirring conditions to obtain reproducible results was recognized [3]. In comparative studies, the existence of the aqueous boundary layers, although acknowledged, was sometimes virtually ignored because of its uniformity throughout a strictly designed and controlled steady-state experimentation work [4-7].

Díez-Sales et al. performed in vitro permeability studies using a series of weakly basic xenobiotics of long-term toxicological interest across a homogeneous lipoidal artificial membrane under several working conditions, including the presence and the partial or total absence of aqueous boundary layers [8]. Synthetic surfactants, at either the critical micelle or supramicellar concentration, were reported to disrupt the in vivo aqueous stagnant diffusion layers adjacent to the membrane at its luminal side, as well as to increase the intrinsic membrane polarity through a direct penetration effect [9-12], and it was thought to evaluate these properties on in vitro artificial membranes. To achieve this, Díez-Sales et al. established permeability-lipophilicity correlations, and compared them for each condition according to previously reported discriminative equations [13-17].

Percutaneous penetration depends essentially on the lipophilicity of the tested substances [18]. The use of homologous series of compounds made it possible to establish behaviour models, which allow predicting the percutaneous absorption capacity of chemically related substances $[19,20]$. Much of the theoretical background information on percutaneous absorption was developed from studies of non-electrolytic permeating species [21,22], and only a little is available on the effect of $p \mathrm{H}$ and $p \mathrm{~K}_{\mathrm{a}}$ on permeation of ionizable drugs through the skin. The vehicle's $p \mathrm{H}$ may have a profound influence upon the percutaneous delivery from topical products [23]. Depending on the $p \mathrm{~K}_{\mathrm{a}}$ of the compound and on the $p \mathrm{H}$ of the vehicle, an equilibrium mixture of ionized and unionized species would be present in the immediate vicinity of the skin [24]. According to the $\mathrm{pH}$-partition simple hypothesis, only the non-ionized forms of drugs are able to pass through lipoidal membranes in significant amounts. However, the permeation of ionized drugs through the skin is possible and therefore must be taken into account, although ionization reduces topical availability [25]. The mechanism by which percutaneous penetration enhancers operate is not fully understood, but the structured lipids within the intercellular channels play an important role in controlling absorption. Penetration enhancers may act by either interacting with the highly ordered lipid structure, or modifying the partitioning of the drug into the tissue [26,27].

In earlier publications, topological charge-transfer (CT) indices were applied to the calculation of the molecular dipole moment of hydrocarbons [28], valence-isoelectronic series of benzene, styrene [29,30] and cyclopentadiene [31], and phenyl alcohols [32]. An index inspired by biological plastic evolution [33,34] and fractal dimension analysis [35,36] improved the results. In the present study, the valence CT indices have been applied to the calculation of the dipole moment of phenyl alcohols, 4-alkylanilines and aliphatic amines. The next section presents the CT indices. Following that, the indices are generalized for heteroatoms. Next, the calculation results are discussed. The last section summarizes the conclusions. 


\section{Materials and methods}

The most important matrices that delineate the labelled chemical graph are the adjacency (A) [37] and the distance (D) matrices, wherein $D_{i j}={ }_{i j}$ if $i=j$, " 0 " otherwise; $\quad{ }_{i j}$ is the shortest edge count between vertices $i$ and $j$ [38]. In $\mathbf{A}, A_{i j}=1$ if vertices $i$ and $j$ are adjacent, " 0 " otherwise. The $\mathbf{D}^{[-2]}$ matrix is that whose elements are the squares of the reciprocal distances $D_{i j}{ }^{-2}$. The intermediate matrix $\mathbf{M}$ is defined as the matrix product of $\mathbf{A}$ by $\mathbf{D}^{[-2]}$ :

$$
\mathbf{M}=\mathbf{A D}^{[-2]}
$$

The CT matrix $\mathbf{C}$ is defined as $\mathbf{C}=\mathbf{M}-\mathbf{M}^{\mathrm{T}}$, where $\mathbf{M}^{\mathrm{T}}$ is the transpose of $\mathbf{M}$ [39]. By agreement $C_{i i}=M_{i i}$. For $i \neq j$, the $C_{i j}$ terms represent a measure of the intramolecular net charge transferred from atom $j$ to $i$. The topological $C T$ indices $G_{k}$ are described as the sum of absolute values of the $C_{i j}$ terms defined for the vertices $i, j$ placed at a topological distance $D_{i j}$ equal to $k$ :

$$
G_{k}=\sum_{i=1}^{N-1} \sum_{j=i+1}^{N}\left|C_{i j}\right| \delta\left(k, D_{i j}\right)
$$

where $N$ is the number of vertices in the graph, $D_{i j}$ are the entries of the $\mathbf{D}$ matrix, and $\delta$ is the Kronecker $\delta$ function, being $\delta=1$ for $i=j$ and $\delta=0$ for $i \neq j . G_{k}$ represents the sum of all the $C_{i j}$ terms, for every pair of vertices $i$ and $j$ at topological distance $k$. Other topological CT index, $J_{k}$, is defined as:

$J_{k}=\frac{G_{k}}{N-1}$

This index represents the mean value of CT for each edge, since the number of edges for acyclic compounds is $N-1$.

The algebraic semisum $C T$ index $\mu_{\mathrm{alg}}$ is defined as

$$
\mu_{a \mathrm{~g}}=\frac{1}{2} \sum_{i=1}^{N-1} \sum_{j=i+1}^{N} A_{i j}\left|C_{i j}\right|=\frac{1}{2} \sum_{e=1}^{m}\left|C^{e}\right|
$$

where $C^{e}$ is $C_{i j}$ index for vertices $i$ and $j$ connected by edge $e$ [28]. The sum extends for all pairs of adjacent vertices in the molecular graph and $\mu_{\text {alg }}$ is a graph invariant. An edge analysis suggests that each dipole moment $\mu^{e}$, of edge $e$ connecting vertices $i$ and $j$ can be evaluated from its edge $C^{e}$ index as:

$$
\mu^{e}=\frac{1}{2} C^{e}
$$

Each edge dipole can be associated with a vector $\mu^{\mathrm{e}}$ in space. $\mu^{\mathrm{e}}$ has magnitude $\left|\mu^{e}\right|$, lies in the edge $e$ connecting vertices $i$ and $j$, and its direction is $j \rightarrow i$. The molecular dipole vector $\mu$ results as the vector sum of the edge dipoles as:

$\boldsymbol{\mu}=\sum_{e=1}^{m} \boldsymbol{\mu}^{e}=\frac{1}{2} \sum_{e=1}^{m} \mathbf{C}^{e}$

summed for all the $m$ edges in the molecular graph. The vector semisum $C T$ index $\mu_{\mathrm{vec}}$ is defined as the module of $\mu$ :

$$
\mu_{\mathrm{vec}}=N(\mu)=\left(\mu_{x}^{2}+\mu_{y}^{2}+\mu_{z}^{2}\right)^{1 / 2}
$$

Therefore, $\mu_{\mathrm{vec}}$ is a graph invariant. 


\section{Valence charge-transfer indices for heteroatoms}

When heteroatoms are present, some way of discriminating atoms of different kinds needs to be considered [40]. In valence CT indices terms, the presence of each heteroatom is taken into account by introducing its electronegativity value in the corresponding entry of the main diagonal of the adjacency matrix A. For each heteroatom $X$, its entry $A_{i i}$ is redefined as:

$A_{i i}^{V}=2.2\left(\chi_{X}-\chi_{\mathrm{C}}\right)$

to give the valence adjacency $\mathbf{A}^{V}$ matrix, where $\chi_{X}$ and $\chi_{\mathrm{C}}$ are the electronegativities of heteroatom $X$ and carbon, respectively, in Pauling units. The subtractive term keeps $A_{i i}{ }^{V}=0$ for the $\mathrm{C}$ atom, and the factor gives $A_{i i}{ }^{V}=2.2$ for $\mathrm{O}$, which was taken as standard. From $\mathbf{A}^{V}, \mathbf{M}^{V}$ and $\mathbf{C}^{V}, G_{k}{ }^{V}, J_{k}{ }^{V}, \mu_{\mathrm{alg}}{ }^{V}$ and $\mu_{\mathrm{vec}}{ }^{V}$ are calculated following the former procedure with $\mathbf{A}^{V} . C_{i i}{ }^{V}, G_{k}{ }^{V}, J_{k}{ }^{V}, \mu_{\mathrm{alg}}{ }^{V}$ and $\mu_{\mathrm{vec}}{ }^{V}$ are graph invariants. $\mu_{\mathrm{vec}}$ is sensitive only to the steric effect of heteroatoms, while $\mu_{\mathrm{vec}}{ }^{V}$ is sensitive to both electronic and steric effects.

\section{Calculation Rresults and Discussion}

The molecular CT indices $G_{k}, J_{k}, G_{k}^{V}$ and $J_{k}^{V}$ (with $k<6$ ) are reported in Table 1 for a series of 13 phenyl alcohols (eight form a homologous series and five are congeneric) and 14 amines (seven homologous 4-alkylanilines and seven congeneric). In particular, for the homologous phenyl alcohols, $G_{1}$ and $G_{2}$ are sensitive to the presence of the alkyl chain, and $G_{3}, G_{4}$ and $G_{5}$ indicate, respectively, the occurrence of at least 2, 3 and $4 \mathrm{C}$ atoms in this chain. For the 4-alkylanilines, $G_{1}$ is a sign of the incidence of the alkyl chain, and $G_{2}-G_{5}$ signify the existence of at least 2-5 C atoms in this chain. For both series, $\mathrm{G}_{1}{ }^{V}$ denotes the presence of the alkyl chain, and $\mathrm{G}_{2}{ }^{V}-\mathrm{G}_{5}{ }^{V}$ imply the occurrence of at least $2-5 \mathrm{C}$ atoms in this chain.

Table 1. Values of the $G_{k}$ and $J_{k}$ charge-transfer indices up to fifth order for phenyl alcohols or amines

\begin{tabular}{lcccccc}
\hline Molecule & $\boldsymbol{N}$ & $\boldsymbol{G}_{\mathbf{1}}$ & $\boldsymbol{G}_{\mathbf{2}}$ & $\boldsymbol{G}_{\mathbf{3}}$ & $\boldsymbol{G}_{\mathbf{4}}$ & $\boldsymbol{G}_{\mathbf{5}}$ \\
\hline phenol & 7 & 2.0000 & 0.8889 & 0.3750 & 0.2222 & 0.0000 \\
benzyl alcohol & 8 & 1.2500 & 6.7778 & 0.8125 & 0.4133 & 0.1250 \\
2-phenyl-1-ethanol & 9 & 1.2500 & 6.7778 & 0.8750 & 0.5644 & 0.2431 \\
3-phenyl-1-propanol & 10 & 1.2500 & 6.7778 & 0.8750 & 0.6044 & 0.3333 \\
4-phenyl-1-butanol & 11 & 1.2500 & 6.7778 & 0.8750 & 0.6044 & 0.3611 \\
5-phenyl-1-pentanol & 12 & 1.2500 & 6.7778 & 0.8750 & 0.6044 & 0.3611 \\
6-phenyl-1-hexanol & 13 & 1.2500 & 6.7778 & 0.8750 & 0.6044 & 0.3611 \\
7-phenyl-1-heptanol & 14 & 1.2500 & 6.7778 & 0.8750 & 0.6044 & 0.3611 \\
1-phenyl-2-propanol & 10 & 2.2500 & 6.7778 & 0.9375 & 0.7156 & 0.3611 \\
2-phenyl-2-propanol & 10 & 2.7500 & 7.2222 & 1.5625 & 0.7956 & 0.3750 \\
3-phenyl-2-propen-1-ol & 10 & 1.2500 & 8.2222 & 0.8750 & 0.4844 & 0.2708 \\
1-phenyl-1-pentanol & 12 & 1.7500 & 7.1111 & 1.3125 & 0.8756 & 0.4861 \\
1-phenyl-2-pentanol & 12 & 2.2500 & 7.0000 & 1.0625 & 0.7556 & 0.4792 \\
aniline & 7 & 1.2500 & 6.6667 & 0.5000 & 0.2222 & 0.0000 \\
4-methylaniline & 8 & 2.5000 & 8.0000 & 0.8750 & 0.5244 & 0.0000 \\
4-ethylaniline & 9 & 2.5000 & 8.1111 & 1.1875 & 0.7156 & 0.1806 \\
4-propylaniline & 10 & 2.5000 & 8.1111 & 1.2500 & 0.8667 & 0.2986 \\
\hline
\end{tabular}


Table 1. Cont.

\begin{tabular}{|c|c|c|c|c|c|c|}
\hline Molecule & $N$ & $G_{1}$ & $G_{2}$ & $G_{3}$ & $G_{4}$ & $G_{5}$ \\
\hline 4-butylaniline & 11 & 2.5000 & 8.1111 & 1.2500 & 0.9067 & 0.3889 \\
\hline 4-pentylaniline & 12 & 2.5000 & 8.1111 & 1.2500 & 0.9067 & 0.4167 \\
\hline 4-hexylaniline & 13 & 2.5000 & 8.1111 & 1.2500 & 0.9067 & 0.4167 \\
\hline 2-methylaniline & 8 & 2.0000 & 8.2222 & 1.0000 & 0.4444 & 0.0000 \\
\hline 4-isopropylaniline & 10 & 3.0000 & 8.2222 & 1.5000 & 0.9067 & 0.3611 \\
\hline 4-(1'-propenyl)aniline & 10 & 2.5000 & 9.4444 & 1.0000 & 0.7467 & 0.2639 \\
\hline 1-aminonaphthalene & 11 & 1.7500 & 12.2222 & 1.6875 & 0.9822 & 0.1250 \\
\hline 2-aminonaphthalene & 11 & 2.2500 & 12.0000 & 1.5000 & 0.9911 & 0.1736 \\
\hline 4-cyclohexylaniline & 13 & 2.5000 & 8.2222 & 1.5625 & 1.0578 & 0.5208 \\
\hline 4-aminobiphenyl & 13 & 2.2500 & 13.7778 & 1.2500 & 0.7911 & 0.4167 \\
\hline Molecule & $J_{1}$ & $J_{2}$ & & $J_{3}$ & $J_{4}$ & $J_{5}$ \\
\hline phenol & 0.3333 & 0.1481 & & 0.0625 & 0.0370 & 0.0000 \\
\hline benzyl alcohol & 0.1786 & 0.9683 & & 0.1161 & 0.0590 & 0.0179 \\
\hline 2-phenyl-1-ethanol & 0.1563 & 0.8472 & & 0.1094 & 0.0706 & 0.0304 \\
\hline 3-phenyl-1-propanol & 0.1389 & 0.7531 & & 0.0972 & 0.0672 & 0.0370 \\
\hline 4-phenyl-1-butanol & 0.1250 & 0.6778 & & 0.0875 & 0.0604 & 0.0361 \\
\hline 5-phenyl-1-pentanol & 0.1136 & 0.6162 & & 0.0795 & 0.0549 & 0.0328 \\
\hline 6-phenyl-1-hexanol & 0.1042 & 0.5648 & & 0.0729 & 0.0504 & 0.0301 \\
\hline 7-phenyl-1-heptanol & 0.0962 & 0.5214 & & 0.0673 & 0.0465 & 0.0278 \\
\hline 1-phenyl-2-propanol & 0.2500 & 0.7531 & & 0.1042 & 0.0795 & 0.0401 \\
\hline 2-phenyl-2-propanol & 0.3056 & 0.8025 & & 0.1736 & 0.0884 & 0.0417 \\
\hline 3-phenyl-2-propen-1-ol & 0.1389 & 0.9136 & & 0.0972 & 0.0538 & 0.0301 \\
\hline 1-phenyl-1-pentanol & 0.1591 & 0.6465 & & 0.1193 & 0.0796 & 0.0442 \\
\hline 1-phenyl-2-pentanol & 0.2045 & 0.6364 & & 0.0966 & 0.0687 & 0.0436 \\
\hline aniline & 0.2083 & 1.1111 & & 0.0833 & 0.0370 & 0.0000 \\
\hline 4-methylaniline & 0.3571 & 1.1429 & & 0.1250 & 0.0749 & 0.0000 \\
\hline 4-ethylaniline & 0.3125 & 1.0139 & & 0.1484 & 0.0894 & 0.0226 \\
\hline 4-propylaniline & 0.2778 & 0.9012 & & 0.1389 & 0.0963 & 0.0332 \\
\hline 4-butylaniline & 0.2500 & 0.8111 & & 0.1250 & 0.0907 & 0.0389 \\
\hline 4-pentylaniline & 0.2273 & 0.7374 & & 0.1136 & 0.0824 & 0.0379 \\
\hline 4-hexylaniline & 0.2083 & 0.6759 & & 0.1042 & 0.0756 & 0.0347 \\
\hline 2-methylaniline & 0.2857 & 1.1746 & & 0.1429 & 0.0635 & 0.0000 \\
\hline 4-isopropylaniline & 0.3333 & 0.9136 & & 0.1667 & 0.1007 & 0.0401 \\
\hline 4-(1'-propenyl)aniline & 0.2778 & 1.0494 & & 0.1111 & 0.0830 & 0.0293 \\
\hline 1-aminonaphthalene & 0.1750 & 1.2222 & & 0.1688 & 0.0982 & 0.0125 \\
\hline 2-aminonaphthalene & 0.2250 & 1.2000 & & 0.1500 & 0.0991 & 0.0174 \\
\hline 4-cyclohexylaniline & 0.2083 & 0.6852 & & 0.1302 & 0.0881 & 0.0434 \\
\hline 4-aminobiphenyl & 0.1875 & 1.1481 & & 0.1042 & 0.0659 & 0.0347 \\
\hline
\end{tabular}


Table 1. Cont.

\begin{tabular}{lccccc}
\hline Molecule & $\boldsymbol{G}_{\mathbf{1}}{ }^{\boldsymbol{V}}$ & $\boldsymbol{G}_{\mathbf{2}}{ }^{\boldsymbol{V}}$ & $\boldsymbol{G}_{\mathbf{3}}{ }^{\boldsymbol{V}}$ & $\boldsymbol{G}_{\mathbf{4}}{ }^{\boldsymbol{V}}$ & $\boldsymbol{G}_{\mathbf{5}}{ }^{\boldsymbol{V}}$ \\
\hline phenol & 2.2000 & 1.1000 & 0.3639 & 0.0847 & 0.0000 \\
benzyl alcohol & 2.9500 & 6.6611 & 0.5625 & 0.2533 & 0.0370 \\
2-phenyl-1-ethanol & 2.9500 & 7.1056 & 0.7444 & 0.4044 & 0.1319 \\
3-phenyl-1-propanol & 2.9500 & 7.1056 & 0.9944 & 0.5019 & 0.2222 \\
4-phenyl-1-butanol & 2.9500 & 7.1056 & 0.9944 & 0.6619 & 0.2824 \\
5-phenyl-1-pentanol & 2.9500 & 7.1056 & 0.9944 & 0.6619 & 0.3936 \\
6-phenyl-1-hexanol & 2.9500 & 7.1056 & 0.9944 & 0.6619 & 0.3936 \\
7-phenyl-1-heptanol & 2.9500 & 7.1056 & 0.9944 & 0.6619 & 0.3936 \\
1-phenyl-2-propanol & 3.4500 & 7.6556 & 0.8069 & 0.5556 & 0.2500 \\
2-phenyl-2-propanol & 3.4500 & 8.2056 & 1.3125 & 0.6356 & 0.2870 \\
3-phenyl-2-propen-1-ol & 2.9500 & 8.3278 & 0.6306 & 0.3819 & 0.1597 \\
1-phenyl-1-pentanol & 2.9500 & 7.3222 & 1.1819 & 0.7731 & 0.4861 \\
1-phenyl-2-pentanol & 3.4500 & 7.6556 & 1.0514 & 0.7331 & 0.3681 \\
aniline & 0.8500 & 6.2222 & 0.2556 & 0.1535 & 0.0000 \\
4-methylaniline & 2.1000 & 7.5556 & 0.6306 & 0.4557 & 0.0440 \\
4-ethylaniline & 2.1000 & 7.6667 & 0.9431 & 0.6468 & 0.1690 \\
4-propylaniline & 2.1000 & 7.6667 & 1.0056 & 0.7979 & 0.2871 \\
4-butylaniline & 2.1000 & 7.6667 & 1.0056 & 0.8379 & 0.3773 \\
4-pentylaniline & 2.1000 & 7.6667 & 1.0056 & 0.8379 & 0.4051 \\
4-hexylaniline & 2.1000 & 7.6667 & 1.0056 & 0.8379 & 0.4051 \\
2-methylaniline & 1.6000 & 7.7778 & 0.8778 & 0.3757 & 0.0000 \\
4-isopropylaniline & 2.6000 & 7.7778 & 1.2556 & 0.8379 & 0.3171 \\
4-(1'-propenyl)aniline & 2.1000 & 9.0000 & 0.7556 & 0.6779 & 0.2199 \\
1-aminonaphthalene & 1.3500 & 11.6722 & 1.3208 & 0.7760 & 0.0810 \\
2-aminonaphthalene & 1.8500 & 11.5556 & 1.2556 & 0.8536 & 0.0856 \\
4-cyclohexylaniline & 2.1000 & 7.7778 & 1.3181 & 0.9890 & 0.4768 \\
4-aminobiphenyl & 1.8500 & 13.3333 & 1.0056 & 0.7224 & 0.3727 \\
\hline
\end{tabular}

\begin{tabular}{lccccc}
\hline Molecule & $\boldsymbol{J}_{\mathbf{1}}{ }^{\boldsymbol{V}}$ & $\boldsymbol{J}_{\mathbf{2}}{ }^{\boldsymbol{V}}$ & $\boldsymbol{J}_{\mathbf{3}}{ }^{\boldsymbol{V}}$ & $\boldsymbol{J}_{\mathbf{4}}{ }^{\boldsymbol{V}}$ & $\boldsymbol{J}_{\mathbf{5}}{ }^{\boldsymbol{V}}$ \\
\hline phenol & 0.3637 & 0.1833 & 0.0606 & 0.0141 & 0.0000 \\
benzyl alcohol & 0.4214 & 0.9516 & 0.0804 & 0.0362 & 0.0053 \\
2-phenyl-1-ethanol & 0.3688 & 0.8882 & 0.0931 & 0.0506 & 0.0165 \\
3-phenyl-1-propanol & 0.3278 & 0.7895 & 0.1105 & 0.0558 & 0.0247 \\
4-phenyl-1-butanol & 0.2950 & 0.7106 & 0.0994 & 0.0662 & 0.0282 \\
5-phenyl-1-pentanol & 0.2682 & 0.6460 & 0.0904 & 0.0602 & 0.0358 \\
6-phenyl-1-hexanol & 0.2458 & 0.5921 & 0.0829 & 0.0552 & 0.0328 \\
7-phenyl-1-heptanol & 0.2269 & 0.5466 & 0.0765 & 0.0509 & 0.0303 \\
1-phenyl-2-propanol & 0.3833 & 0.8506 & 0.0897 & 0.0617 & 0.0278 \\
2-phenyl-2-propanol & 0.3833 & 0.9117 & 0.1458 & 0.0706 & 0.0319 \\
3-phenyl-2-propen-1-ol & 0.3278 & 0.9253 & 0.0701 & 0.0424 & 0.0177 \\
\hline
\end{tabular}


Table 1. Cont.

\begin{tabular}{lccccc}
\hline Molecule & $\boldsymbol{J}_{\mathbf{1}}{ }^{\boldsymbol{N}}$ & $\boldsymbol{J}_{\mathbf{2}}{ }^{\boldsymbol{V}}$ & $\boldsymbol{J}_{\mathbf{3}}{ }^{\boldsymbol{V}}$ & $\boldsymbol{J}_{\mathbf{4}}{ }^{\boldsymbol{V}}$ & $\boldsymbol{J}_{\mathbf{5}}{ }^{\boldsymbol{V}}$ \\
\hline 1-phenyl-1-pentanol & 0.2682 & 0.6657 & 0.1074 & 0.0703 & 0.0442 \\
1-phenyl-2-pentanol & 0.3136 & 0.6960 & 0.0956 & 0.0666 & 0.0335 \\
aniline & 0.1417 & 1.0370 & 0.0426 & 0.0256 & 0.0000 \\
4-methylaniline & 0.3000 & 1.0794 & 0.0901 & 0.0651 & 0.0063 \\
4-ethylaniline & 0.2625 & 0.9583 & 0.1179 & 0.0809 & 0.0211 \\
4-propylaniline & 0.2333 & 0.8519 & 0.1117 & 0.0887 & 0.0319 \\
4-butylaniline & 0.2100 & 0.7667 & 0.1006 & 0.0838 & 0.0377 \\
4-pentylaniline & 0.1909 & 0.6970 & 0.0914 & 0.0762 & 0.0368 \\
4-hexylaniline & 0.1750 & 0.6389 & 0.0838 & 0.0698 & 0.0338 \\
2-methylaniline & 0.2286 & 1.1111 & 0.1254 & 0.0537 & 0.0000 \\
4-isopropylaniline & 0.2889 & 0.8642 & 0.1395 & 0.0931 & 0.0352 \\
4-(1'-propenyl)aniline & 0.2333 & 1.0000 & 0.0840 & 0.0753 & 0.0244 \\
1-aminonaphthalene & 0.1350 & 1.1672 & 0.1321 & 0.0776 & 0.0081 \\
2-aminonaphthalene & 0.1850 & 1.1556 & 0.1256 & 0.0854 & 0.0086 \\
4-cyclohexylaniline & 0.1750 & 0.6481 & 0.1098 & 0.0824 & 0.0397 \\
4-aminobiphenyl & 0.1542 & 1.1111 & 0.0838 & 0.0602 & 0.0311 \\
\hline
\end{tabular}

The molecular dipole moments $\mu$ (experimental, and calculated as vector semisums of $C_{i j}$ and $C_{i j}{ }^{V}$ ) are listed in Table 2. As experimental values were not available for any whole series, some reference values were computed with program MOPAC-AM1. The reliability of AM1 results was tested with all the entries in Table 2 for which experimental data were available. AM1 calculations adequately reproduced the oscillatory behaviour of the experimental data, mimicking four minima (phenol, 2-phenyl-1-ethanol, aniline and 4-ethylaniline) and four maxima (benzyl alcohol, 3-phenyl-1-propanol, 4-methylaniline and 4-propylaniline). This test suggested that AM1 allows a good approximation, at least for the general performance of both homologous series, and that the error is sufficiently constant throughout the series. The number of compounds in both series was not increased because longer molecules are not percutaneous enhancers owing to their low transdermal penetration.

Table 2. Molecular dipole moment values, $\mu$ (D), for alcohols and amines calculated with charge-transfer indices.

\begin{tabular}{lcccc}
\hline Molecule & $\begin{array}{c}\text { Number } \\
\text { atoms in alkyl chain }\end{array}$ & $\begin{array}{c}\text { Vector } \\
\text { semisum }\end{array}$ & $\begin{array}{c}\text { Valence vector }^{\text {cemisum }} \\
\text { semperimental }\end{array}$ & \\
\hline phenol & 0 & 0.737 & 2.431 & $1.400\left(1.233^{\mathrm{b}}\right)$ \\
benzyl alcohol & 1 & 0.589 & 2.487 & $1.700\left(1.568^{\mathrm{b}}\right)$ \\
2-phenyl-1-ethanol & 2 & 0.700 & 2.257 & $1.590\left(1.497^{\mathrm{b}}\right)$ \\
3-phenyl-1-propanol & 3 & 0.573 & 2.519 & $1.640\left(1.597^{\mathrm{b}}\right)$ \\
4-phenyl-1-butanol & 4 & 0.702 & 2.249 & $1.345^{\mathrm{b}}$ \\
5-phenyl-1-pentanol & 5 & 0.573 & 2.519 & $1.626^{\mathrm{b}}$ \\
\hline
\end{tabular}


Table 2. Cont.

\begin{tabular}{lcccl} 
Molecule & $\begin{array}{c}\text { Number } \\
\text { atoms in alkyl chain }\end{array}$ & $\begin{array}{c}\text { Vector } \\
\text { semisum }\end{array}$ & $\begin{array}{c}\text { Valence vector } \\
\text { semisum }\end{array}$ & Experimental $^{\mathrm{a}}$ \\
6-phenyl-1-hexanol & 6 & 0.702 & 2.250 & $1.346^{\mathrm{b}}$ \\
7-phenyl-1-heptanol & 7 & 0.573 & 2.518 & $1.634^{\mathrm{b}}$ \\
1-phenyl-2-propanol & 3 & 0.923 & 2.347 & $1.564^{\mathrm{b}}$ \\
2-phenyl-2-propanol & 3 & 0.780 & 2.821 & $1.463^{\mathrm{b}}$ \\
3-phenyl-2-propen-1-ol & 3 & 0.561 & 2.495 & $1.591^{\mathrm{b}}$ \\
1-phenyl-1-pentanol & 5 & 0.426 & 2.794 & $1.746^{\mathrm{b}}$ \\
1-phenyl-2-pentanol & 5 & 0.895 & 2.367 & $1.496^{\mathrm{b}}$ \\
aniline & 0 & 0.655 & 0.792 & $1.560\left(1.584^{\mathrm{b}}\right)$ \\
4-methylaniline & 1 & 0.079 & 1.526 & $1.640\left(1.423^{\mathrm{b}}\right)$ \\
4-ethylaniline & 2 & 0.385 & 1.339 & $1.492^{\mathrm{b}}$ \\
4-propylaniline & 3 & 0.087 & 1.533 & $1.502^{\mathrm{b}}$ \\
4-butylaniline & 4 & 0.398 & 1.333 & $1.511^{\mathrm{b}}$ \\
4-pentylaniline & 5 & 0.093 & 1.533 & $1.489^{\mathrm{b}}$ \\
4-hexylaniline & 6 & 0.395 & 1.332 & $1.496^{\mathrm{b}}$ \\
2-methylaniline & 1 & 1.187 & 0.779 & $1.590\left(1.560^{\mathrm{b}}\right)$ \\
4-isopropylaniline & 3 & 0.625 & 1.490 & $1.450^{\mathrm{b}}$ \\
4-(1'-propenyl)aniline & 3 & 0.108 & 1.512 & $1.512^{\mathrm{b}}$ \\
1-aminonaphthalene & 4 & 0.645 & 0.802 & $1.490\left(1.690^{\mathrm{b}}\right)$ \\
2-aminonaphthalene & 4 & 0.655 & 0.792 & $1.820\left(1.868^{\mathrm{b}}\right)$ \\
4-cyclohexylaniline & 6 & 0.480 & 1.126 & $1.760\left(1.447^{\mathrm{b}}\right)$ \\
4-aminobiphenyl & 6 & 0.651 & 0.795 & $1.447^{\mathrm{b}}$ \\
\hline
\end{tabular}

${ }^{\mathrm{a}}$ Taken from Reference 44.

${ }^{\mathrm{b}}$ Calculations carried out with program MOPAC-AM1.

In particular, 3-phenyl-2-propen-1-ol was chosen in order to compare the influence of a double bond in the alkyl chain region of a phenyl alcohol. The presence of the enol group (conjugated double bond in $\beta$-position with respect to the $-\mathrm{OH}$ group) lent 3-phenyl-2-propen-1-ol to greater rigidity than for 3-phenyl-1-propanol. However, $\mu_{\mathrm{AM} 1}$ change was $<1 \%$. For the three phenyl propanols (primary, secondary and tertiary alcohols), the primary alcohol 3-phenyl-1-propanol showed the greatest $\mu_{\mathrm{AM} 1}$. For the three phenyl pentanols (primary, and $\alpha$ - and $\beta$-phenyl -OH secondary alcohols), the primary alcohol 5-phenyl-1-pentanol presented a relatively large $\mu_{\mathrm{AM} 1}$. On going from 4-propylaniline to 4 -(1'-propenyl)aniline, the effect of the conjugated double bond on $\mu_{\mathrm{AM} 1}$ was again $<1 \%$. For 2 - and 4-methylaniline, the para-(4)-methylaniline showed the greatest $\mu_{\text {experiment }}$ but the smallest $\mu_{\mathrm{AM} 1}$. For 4-propylaniline and 4-isopropylaniline, the more linear 4-propylaniline revealed the greatest $\mu_{\mathrm{AM} 1}$. For 4-butylaniline and both aminonaphthalenes, the rigid 2-aminonaphthalene displayed the greatest $\mu_{\mathrm{AM} 1}$. For 4-hexylaniline, 4-cyclohexylaniline and 4-aminobiphenyl, the more linear 4-hexylaniline exhibited the greatest $\mu_{\mathrm{AM} 1}$. Figure 1 illustrates how the dipole moment of both homologous series fluctuates with the number of $\mathrm{C}$ atoms in the alkyl chain, $n$. For either series, the experimental $\mu$ and calculated $\mu_{\mathrm{vec}}{ }^{V}$ vary together in an alternate fashion: for odd $n, \mu$ is greater than for even $n$. However, the 
computed $\mu_{\mathrm{vec}}$ presents the opposite tendency. The corresponding interpretation is that, as $\mu_{\mathrm{vec}}$ is not sensitive to the electronic effect of either heteroatom, the steric $\left(\mu_{\mathrm{vec}}\right)$ and electronic $\left(\mu_{\mathrm{vec}}{ }^{V}\right)$ factors are antagonistic, and the electronic effect dominates over the steric one. The $\mu_{\text {experiment }}$ decreases $c a .4 \%$ for either $n=0 \rightarrow 6$ or $n=1 \rightarrow 7$.

Figure 1. Dipole moment of phenyl alcohols (empty symbols) and 4-alkylanilines (filled symbols) as a function of the number of $\mathrm{C}$ atoms in alkyl chain.

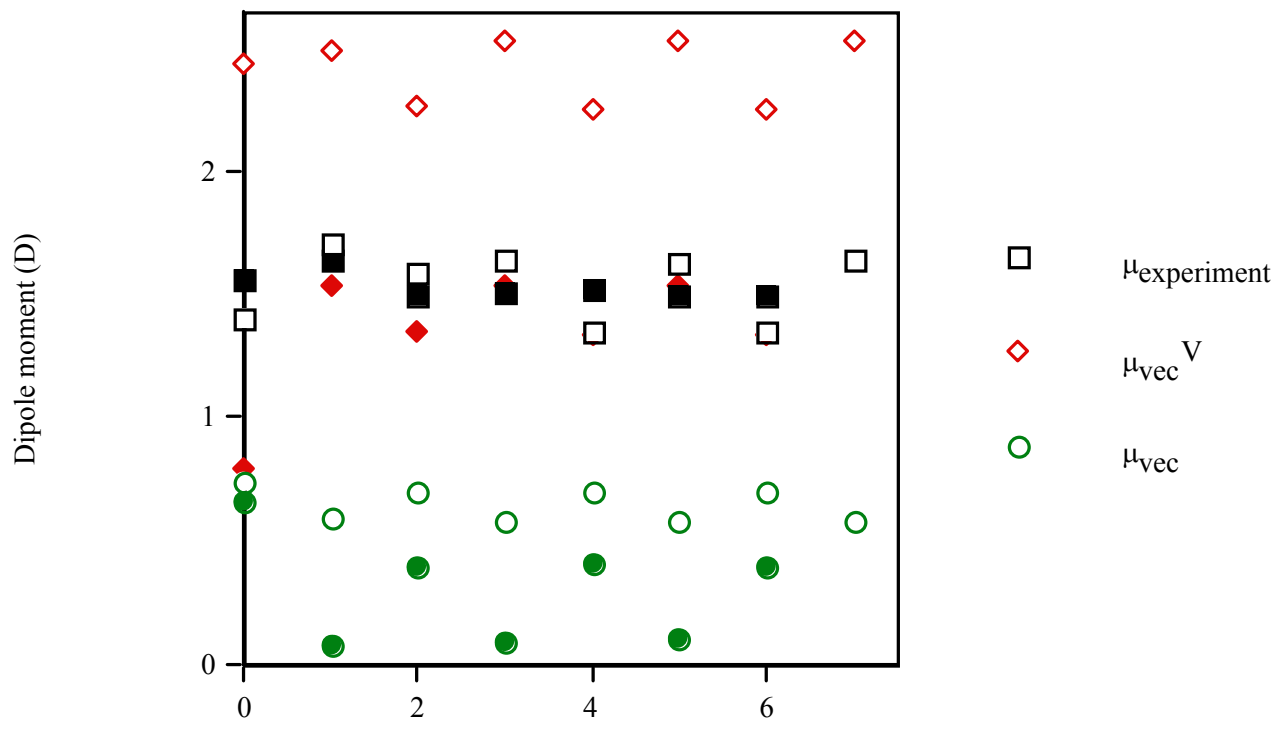

Number of $\mathrm{C}$ atoms in alkyl chain

The experimental dipole moments and CT indices were correlated for the homologous series. As $\mu_{\text {experiment }}$ for the 4-alkylanilines is almost constant $(\approx 1.5 \mathrm{D})$, especially for $n>1$, it was represented by only one point in $\mu$ space. Therefore, the joint fit of phenyl alcohols and this point was performed. The best linear fit turned out to be:

$\mu=1.45+0.0906 G_{2}-9.39 J_{4}^{V}$

$N=9 \quad r=0.688 \quad \mathrm{SD}=0.115 \quad F=2.7 \quad$ MAPE $=4.54 \% \quad$ AEV $=0.5269$

where MAPE is the mean absolute percentage error and AEV is the approximation error variance. All other models with greater MAPE and AEV were discarded, including those models counting the number of $\mathrm{C}$ atoms in the chain. The variables $G_{2}$ and $J_{4}{ }^{V}$ suggest the importance of electronic density distribution in the different compounds. The best quadratic model for $\mu$ turned out to be:

$\mu=17.0-12.8 G_{1}^{V}+2.60\left(G_{1}^{V}\right)^{2}-97.8\left(J_{4}^{V}\right)^{2}$

$N=9 \quad \mathrm{MAPE}=4.12 \%$

$\mathrm{AEV}=0.5072$

and AEV decreased by $4 \%$. The inclusion of $\mu_{\mathrm{vec}}$ or $\mu_{\mathrm{vec}}{ }^{V}$ did not improve the fit.

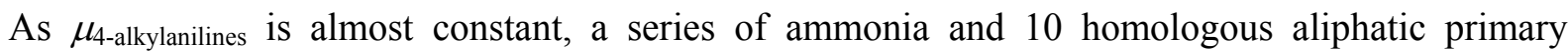
amines was chosen in order to compare the dipole moments [41]. Figure 2 shows how the dipole moment of the aliphatic amine series varies with the number of $\mathrm{C}$ atoms, $n$. In general, $\mu_{\text {experiment }}$ and $\mu_{\mathrm{vec}}{ }^{V}$ change together in an alternate fashion $\left(\mu_{\mathrm{odd} n}>\mu_{\mathrm{even} n}\right)$ while $\mu_{\mathrm{vec}, \text { odd } n}<\mu_{\mathrm{vec}, \text { even } n}$. Again, the 
steric and electronic factors are antagonistic, and the electronic effect dominates. Although $\mu_{\text {experiment }}$ decreases by $9 \%$ for $n=0 \rightarrow 10$, it increases by $21 \%$ for $n=1 \rightarrow 9$.

Figure 2. Dipole moment of ammonia and aliphatic amines as a function of the number of $\mathrm{C}$ atoms, $n$. Point $n=9$ is AM1 calculation.

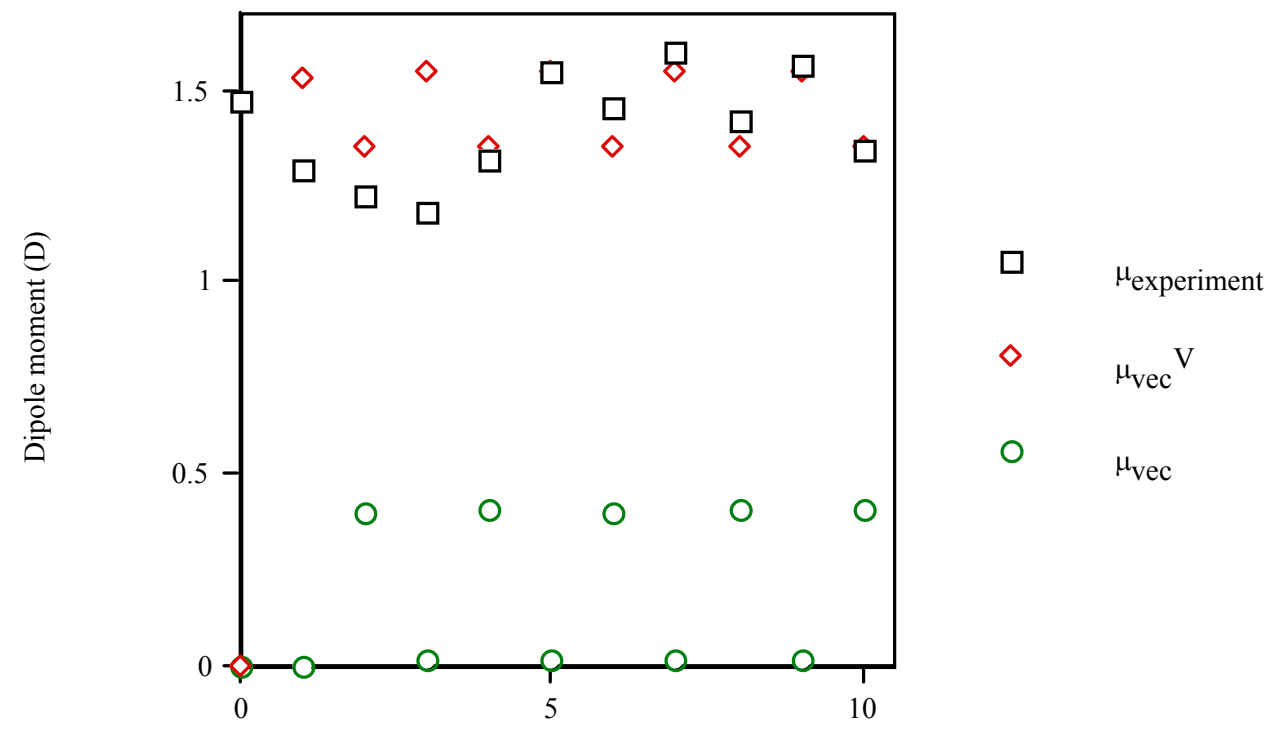

Number of $\mathrm{C}$ atoms

As $\mu_{4 \text {-alkylanilines }}$ hardly varies, it represents only one point in the space of $\mu$ and the joint fit of the aliphatic amines and this point was performed. The best linear model for $\mu$ turned out to be:

$\mu=1.45-0.285 G_{1}-0.162 J_{1}^{V}+32.6 J_{5}^{V}$

$N=12 \quad r=0.879 \quad \mathrm{SD}=0.078 \quad F=9.1 \quad$ MAPE $=3.60 \% \quad$ AEV $=0.2291$

and AEV decreased by $57 \%$. The best quadratic model and the inclusion of $\mu_{\mathrm{vec}}$ or $\mu_{\mathrm{vec}}{ }^{V} \operatorname{did}$ not improve the correlation.

A comparative study was performed for each homologous series. For ammonia and the aliphatic amines, the best linear model for $\mu$ turned out to be:

$$
\begin{array}{lrrr}
\mu=1.46-0.305 J_{1}+2.17 J_{3}-0.162 G_{1}^{V}+30.8 J_{5}^{V} & & \\
N=11 & r=0.883 \quad \mathrm{SD}=0.086 & F=5.3 \quad \text { MAPE }=3.59 \% \quad \text { AEV }=0.2226
\end{array}
$$

and AEV decreased by $58 \%$ from that in Equation (6).

No superposition of the corresponding $G_{k}-J_{k}$ or $G_{k}{ }^{V}-J_{k}{ }^{V}$ pairs is observed in Equations (6-9), which diminishes the risk of co-linearity in the fits, given the close relationship between each pair $G_{k}-J_{k}$ in Equation (2) $[42,43]$.

If the first point in Figure 2 (Equation 9) is omitted the best linear fit turns out to be:

$$
\begin{array}{lrrr}
\mu & =1.29+1.27 G_{3}-2290 J_{4}-0.602 G_{2}^{V}+0.584 J_{2}^{V}+4190 J_{5}^{V} \\
N=10 & r=0.950 \quad \mathrm{SD}=0.069 \quad F=7.3 \quad \text { MAPE }=1.92 \% \quad \text { AEV }=0.1004
\end{array}
$$


and AEV decreased $81 \%$. Equation (10) is overfitted but it is given with the only purpose of showing that ammonia $(n=0)$, which is not an aliphatic amine, acts as a marginal outlier for Equation (9). The best quadratic model and the inclusion of $\mu_{\mathrm{vec}}$ or $\mu_{\mathrm{vec}}{ }^{V}$ did not improve the correlation.

For the homologous phenyl alcohols, the best linear model turned out to be:

$$
\begin{array}{lllll}
\mu=-0.799+0.364 J_{2}^{V}+0.871 \mu_{\mathrm{vec}}^{V} & & & \\
N=8 & r=0.930 & \mathrm{SD}=0.064 & F=16.0 & \mathrm{MAPE}=2.59 \%
\end{array} \quad \mathrm{AEV}=0.1351
$$

and AEV decreased by $74 \%$. Equation (11) is overfitted but it is given with the purpose of justifying that $\mu_{4-a l k y l a n i l i n e s}$ were represented by only one point in $\mu$ space in Equations (6-7). The best quadratic model did not improve the fit.

For the homologous 4-alkylanilines, the best linear model turned out to be:

$$
\begin{array}{lrrr}
\mu=8.87-1.28 G_{2}+1.43 G_{1}^{V} & & \\
N=7 \quad r=0.992 & \mathrm{SD}=0.009 & F=118.1 \quad \text { MAPE }=0.32 \% \quad \text { AEV }=0.0167
\end{array}
$$

and AEV decreased by 97\%. Equation (12) is overfitted but it is given with the purpose of showing that $\mu_{\text {phenyl alcohols }}$ are difficult to fit due to their oscillating variation with the number of $\mathrm{C}$ atoms. The best quadratic model and the inclusion of $\mu_{\mathrm{vec}}$ or $\mu_{\mathrm{vec}}{ }^{V}$ did not improve the correlation.

\section{Conclusions}

From the preceding results the following conclusions can be drawn:

1. Inclusion of the heteroatom in the $\pi$-electron system was beneficial for the description of the dipole moment, owing to either the role of the additional $p$ orbitals provided by the heteroatom or the role of steric factors in the $\pi$-electron conjugation. The analysis of the electronic and steric factors in $\mu$ caused by the heteroatom showed that both factors are antagonistic, and that the electronic factor dominates over the steric one.

2. The conjugated double bond in the alkyl chain of 3-phenyl-2-propen-1-ol and 4-(1'-propenyl)aniline lent to more rigid structures with dipole moment variations $<1 \%$.

3. Linear and quadratic correlation models were obtained for the molecular dipole moments of phenyl alcohols, 4-alkylanilines and aliphatic amines. $\mu_{\mathrm{vec}}{ }^{V}$ improved the multivariable regression equations for $\mu$, diminishing the risk of co-linearity in the fit. Improvements in correlation suggest the general applicability of this index.

\section{Acknowledgements}

The author acknowledges financial support from the Spanish MCT (Plan Nacional I+D+I, Project No. BQU2001-2935-C02-01) and Generalitat Valenciana (DGEUI INF01-051 and INFRA03-047, and OCYT GRUPOS03-173).

\section{References}

1. Stehle, R. G.; Higuchi, W. I. In Vitro Model for Transport of Solutes in Three-Phase System II: Experimental Considerations. J. Pharm. Sci. 1972, 61, 1931-1935

2. Lovering, E. G.; Black, D. B. Diffusion Layers Effects on Permeation of Phenylbutazone through 
Polydimethylsiloxane. J. Pharm. Sci. 1974, 63, 1399-1402

3. Barry, B. W. Novel Mechanisms and Devices to Enable Successful Transdermal Drug Delivery. Eur. J. Pharm. Sci. 2001, 14, 101-114

4. Sato, S.; Kim, S. W. Macromolecular Diffusion through Polymer Membranes. Int. J. Pharm. 1984, 22, 229-255

5. Ackermann, C.; Flynn, G. L.; van Wyk, C. J. Percutaneous Absoption of Urea. Int. J. Cosm. Sci. 1985, 7, 251-264

6. Ackermann, C.; Flynn, G. L.; Smith, W. M. Ether-Water Partitioning and Permeability through Nude Mouse Skin in Vitro. II. Hydrocortisone- $n$-alkyl Esters, Alkanols and Hydrophilic Compounds. Int. J. Pharm. 1987, 36, 67-71

7. Ackermann, C.; Flynn, G. L. Ether-Water Partitioning and Permeability through Nude Mouse Skin in Vitro. I. Urea, Thiourea, Glycerol and Glucose. Int. J. Pharm. 1987, 36, 61-66

8. Díez-Sales, O.; Copoví, A.; Casabó, V. G.; Herráez, M. A Modelistic Approach Showing the Importance of the Stagnant Aqueous Layers in in Vitro Diffusion Studies, and in Vitro-in Vivo Correlations. Int. J. Pharm. 1991, 77, 1-11

9. Plá-Delfina, J. M.; Pérez-Buendía, M. D.; Casabó, V. G.; Peris-Ribera, J. E.; Sánchez-Moyano, E.; Martín-Villodre, A. Absorption Partition Relationships for True Homologous Series of Xenobiotics as a Possible Approach to Study Mechanisms of Surfactants in Absorption. I. Aromatic Amines in Rat Colon. Int. J. Pharm. 1987, 37, 49-64

10. Collado, E. F.; Fabra-Campos, S.; Peris-Ribera, J. E.; Casabó, V. G.; Martín-Villodre, A.: PláDelfina, J. M. Absorption Partition Relationships for True Homologous Series of Xenobiotics as a Possible Approach to Study Mechanisms of Surfactants in Absorption. II. Aromatic Amines in Rat Small Intestine. Int. J. Pharm. 1988, 44, 187-196

11. Garrigues, T. M.; Collado, E. F.; Fabra-Campos, S.; Pérez-Buendía, M. D.; Martín-Villodre, A.; Plá-Delfina, J. M. Absorption Partition Relationships for True Homologous Series of Xenobiotics as a Possible Approach to Study Mechanisms of Surfactants in Absorption. III. Aromatic Amines and Cationic Surfactants. Int. J. Pharm. 1989, 57, 189-196

12. Garrigues, T. M., Pérez-Varona, A. T.; Climent, E.; Bermejo, M. V.; Martín-Villodre, A.; PláDelfina, J. M. Gastric Absorption of Acidic Xenobiotics in the Rat: Biophysical Interpretation of an Apparently Atypical Behaviour. Int. J. Pharm. 1990, 64, 127-138

13. Díez-Sales, O.; Guzmán, D.; Cano, D.; Martín, A.; Sánchez, E.; Herráez, M. A Comparative in Vitro Study of Permeability with Different Synthetic and Biological Membranes. Eur. J. Drug Metab. Pharmacokinet. 1991, Spec. No. 3, 441-446

14. Sánchez-Moyano, E.; Seco, C.; Santolaria, A.; Fabra-Campos, S.; Herráez, M.; Matín-Villodre, A. Partition Behavior of Anilines in Bulk-Phase and High-Performance Liquid Chromatographic Systems: Influence on Correlation with Biological Constants. J. Pharm. Sci. 1992, 81, 720-725

15. Díez-Sales, O.; López-Castellano, A.; Maiques-Lacer, F. J.; Herráez-Domínguez, M. An in Vitro Percutaneous Absorption Study of Non-ionic Compounds across Human Skin. Pharmazie 1993, 48, 684-686

16. Díez-Sales, O.; Pérez-Sayas, E.; Martín-Villodre, A.; Herráez-Domínguez, M. The Prediction of Percutaneous Absorption: I. Influence of the Dermis on in Vitro Permeation Models. Int. J. Pharm. 1993, 100, 1-7 
17. Díez-Sales, O.; Watkinson, A. C.; Herráez-Domínguez, M.; Javaloyes, C.; Hadgraft, J. A

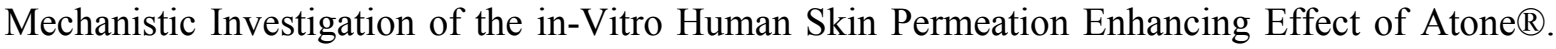
Int. J. Pharm. 1996, 129, 33-40

18. López, A.; Morant, M. J.; Guzmán, D.; Borrás-Blasco, J.; Díez-Sales, O.; Herráez, M. Skin Permeation Model of Phenylalkylcarboxylic Homologous Acids and their Enhancer Effect on Percutaneous Penetration of 5-Fluorouracil. Int. J. Pharm. 1996, 139, 205-213

19. López, A.; Pellett, M. A.; Llinares, F.; Díez-Sales, O.; Herráez, M.; Hadgraft, J. The Enhancer Effect of Several Phenyl Alcohols on Percutaneous Penetration of 5-Fluorouracil. Pharm. Res. 1997, 14, 681-685

20. López, A.; Faus, V.; Díez-Sales, O.; Herráez, M. Skin Permeation Model of Phenyl Alcohols: Comparison of Experimental Conditions. Int. J. Pharm. 1998, 173, 183-191

21. Yalkowsky, S. H.; Flynn, G. L. Transport of Alkyl Homologs across Synthetic and Biological Membranes: A New Model for Chain Length-Activity Relationships. J. Pharm. Sci. 1973, 62, 210-217

22. Flynn, G. L.; Yalkowsky, S. H.; Roseman, T. J. Mass Transport Phenomena and Models: Theoretical Concepts. J. Pharm. Sci. 1974, 63, 479-510

23. Irwin, W. J.; Sanderson, F. D.; Po, A. L. W. Percutaneous Absorption of Ibuprofen: Vehicle Effects on Transport through Rat Skin. Int. J. Pharm. 1990, 66, 193-200

24. Swarbrick, J.; Lee, G.; Brom, J.; Gensmantel, N. P. Drug Permeation through Human Skin II: Permeability of Ionizable Compounds. J. Pharm. Sci. 1984, 73, 1352-1355

25. Banerjee, P. S.; Ritschel, W. A. Transdermal Permeation of Vasopressin. I. Influence of pH, Concentration, Shaving and Surfactant on in Vitro Permeation. Int. J. Pharm. 1989, 49, 189-197

26. Lewis, D.; Hadgraft, J. Mixed Monolayers of Dipalmitoylphosphatidylcholine with Azone or Oleic Acid at the Air-Water Interface. Int. J. Pharm. 1990, 65, 211-218

27. Williams, A. C.; Barry, B. W. Terpenes and the Lipid-Protein Partitioning Theory of Skin Penetration Enhancement. Pharm. Res. 1991, 8, 17-24

28. Torrens, F. A New Topological Index to Elucidate Apolar Hydrocarbons. J. Comput.-Aided Mol. Des. 2001, 15, 709-719

29. Torrens, F. Valence Topological Charge-Transfer Indices for Dipole Moments. J. Mol. Struct. (Theochem) 2003, 621, 37-42

30. Torrens, F. Valence Topological Charge-Transfer Indices for Dipole Moments. Mol. Diversity, in press

31. Torrens, F. Valence Topological Charge-Transfer Indices for Reflecting Polarity: Correction for Heteromolecules. Molecules, submitted for publication

32. Torrens, F. Valence Topological Charge-Transfer Indices for Dipole Moments. Molecules 2003, $8,169-185$

33. Torrens, F. A New Chemical Index Inspired by Biological Plastic Evolution, Indian J. Chem., Sect. A 2003, 42, 1258-1263

34. Torrens, F. A Chemical Index Inspired by Biological Plastic Evolution: Valence-Isoelectronic Series of Aromatics. J. Chem. Inf. Comput. Sci. 2004, 44, 575-581

35. Torrens, F. Fractal Dimension of Transdermal-Delivery Drug Models. Leb. Sci. J., in press

36. Torrens, F. Fractal Dimension of Transdermal-Delivery Drug Models: 4-Alkylanilines. Physica A, submitted for publication 
37. Randić, M. On Characterization of Molecular Branching. J. Am. Chem. Soc. 1975, 97, 6609-6615

38. Hosoya, H. Topological Index. A Newly Proposed Quantity Characterizing the Topological Nature of Structural Isomers of Saturated Hydrocarbons. Bull. Chem. Soc. Jpn. 1971, 44, 23322339

39. Gálvez, J.; García, R.; Salabert, M. T.; Soler, R. Charge Indexes. New Topological Descriptors. J. Chem. Inf. Comput. Sci. 1984, 34, 520-525

40. Kier, L. B.; Hall, L. H. Molecular Connectivity VII: Specific Treatment of Heteroatoms. J. Pharm. Sci. 1976, 65, 1806-1809

41. Lakard, B. Analysis of Permanent Electric Dipole Moments of Aliphatic Amines. Internet Electron. Conference of Molecular Design, 2003

42. Box, G. E. P.; Hunter, W. G.; MacGregor, J. F.; Erjavec, J. Some Problems Associated with the Analysis of Multiresponse Data. Technometrics 1973, 15, 33-51

43. Hocking, R. R. The Analysis and Selection of Variables in Linear Regression. Biometrics 1976, 32, 1-49

44. McClellan, A. L. Tables of Experimental Dipole Moments; Freeman: San Francisco, 1963

(C) 2004 by MDPI (http://www.mdpi.org). Reproduction is permitted for noncommercial purposes. 